\title{
On the Names of the Psychoactive Drugs
}

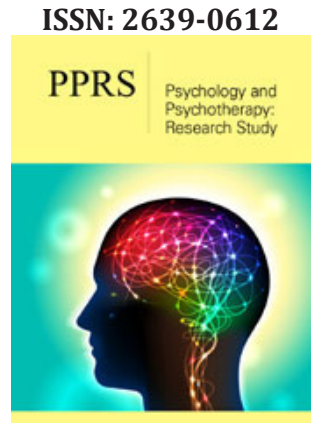

*Corresponding author: Claudio Cabrera Velázquez, Doctor in Psychiatry, Spain

Submission: 漈 July 27, 2019

Published: 想August 07, 2019

Volume 2 - Issue 4

How to cite this article: Claudio Cabrera V. On the Names of the Psychoactive Drugs. Psychol Psychother Res Stud. 2(4). PPRS.000541.2019.

DOI: 10.31031/PPRS.2019.02.000541

Copyright@ Claudio Cabrera Velázquez, This article is distributed under the terms of the Creative Commons Attribution 4.0 International License, which permits unrestricted use and redistribution provided that the original author and source are credited.

\author{
Claudio Cabrera V* \\ Doctor in Psychiatry, Spain
}

\section{Opinion}

Many times the categorical organizations of psychoactive drugs are impractical in the sense that we doctors have a habit, practical and illustrative, of organizing things in discrete categories, which seem to have little to do with the broad spectrum of reality, and much more as regards the issues related to the human mind, among them the psychiatric syndromes. It is common for different syndromes to overlap and present comorbidly: in psychotic cases it is almost the norm to find anxious or depressive conditions, in the most purely depressive cases it is the norm to find anxious or insomnia symptoms. In obsessive conditions it is widely accepted that there is a relevant anxious component almost constantly. In substance abuse disorders it is usual to find another type of symptoms either depressive, anxious or even psychotic: they are presented both previously and as a consequence of substance use. In psychiatry it is normal for syndromes to overlap. Therefore, there are no pure depressive syndromes, nor pure psychotic syndromes, nor pure anxious syndromes, since they almost always occur in symptomatic clusters whose categorization is only indicative for the clinician, but it is not a categorization that really conveys the globality of the case, because each case is broad, complex, multifactorial and unique.

Well, in this context of significant psychiatric pathology is where we make use of psychotropic drugs. The psychoactive drugs, tremendously useful elements in some cases, indispensable in others. It is understood that drugs are also organized into categories: antidepressants, mood stabilizers, antipsychotics, anxiolytics etc. and this is certainly practical for educational and organizational purposes, but in true clinical practice it is a confusing classification, both for general practitioners or other specialties outside of psychiatry as well as for the psychiatrist himself. In an illustrated way, let's start talking about SSRI antidepressants, for example, a large group of medicines called "antidepressants", a name that in my opinion should be changed, because it leads to confusion. It confuses the clinician and confuses the patient, and this ends up implying a misuse of the medication. Why? Because the antidepressant is not only anti-depressant. It has a large number of effects that by calling them antidepressants, they are broadly ignored: for example they are the fundamental treatment of anxious conditions, (and not the so-called anxiolytics, usually referring to benzodiazepines, name also that leads us to equivocation because most of the clinicians indicate them when they see any condition related to anxiety. It is well known that these benzodiazepine medications remain longer than desired in many cases, but the actual treatment of anxiety symptoms is more accurately an SSRI in the vast majority of cases ) Therefore, in this issue of anxious conditions (panic disorder, generalized anxiety disorder, agoraphobia disorder or whatever you want to mention) we must accept that being the SSRI's a most appropriate treatment, calling them antidepressants leads to confusion. And calling benzodiazepines "anxiolytics" invites the clinician to treat these anxious conditions with these benzodiazepine drugs: this leads to confusion and long-term problems. Well, similarly we could make an observation about the so-called antipsychotics. These molecules, undisputed pillars of psychiatric practice since they were discovered in the middle of the last century, have in common that they block the D2 receptors of dopamine in different degrees and places of the CNS, mechanisms in which I will not abound today. But calling them antipsychotic confuses the clinician and the patient. Specifically, I would like to indicate that I find in my clinical practice a huge variety of effects of these so-called antipsychotics that have nothing to do with psychosis. These effects 
are rapid and solid, and often show a capacity for resolving several symptoms that other pharmacological groups hardly offer.

For example, a depressive condition with enormous accompanying anguish, insomnia, rumination, but not psychotic symptoms in any way, benefits greatly from a treatment with an antipsychotic. If we waited for the effect of an antidepressant it could take weeks, but if we combine it with a small amount of a drug from the so-called antipsychotic group, we can find, and in fact, I find in the vast majority of cases, an impressive improvement in 24hours. I speak for example of adding low doses of olanzapine to juggle the discomfort of a depressive condition that should not wait for the mandatory delay of the antidepressant, which takes weeks to show its effects. This particularity of antipsychotics is probably being underused because it is called antipsychotic, a name that leads us to think that these drugs should only be used in psychotic syndromes. And therefore, we are taking little advantage of its capacity, ultimately worsening the patient's prognosis. A wide spectrum of cases of this type can be mentioned: for example, the anti-obsessive effect of the so-called antidepressants, or their own regulatory effect on sleep, or the mood stabilizing effect of the so-called antipsychotics, or the anxiolytic effect of these same antipsychotics. Therefore, it seems that there are beneficial effects of these medications that are being underused and in part it could be thought that it is by the name that they are commonly given in the medical world. It could also be thought that there are undesirable effects of certain drugs due to their overuse in certain conditions and this could be partly due to the denomination made of them (for example, anxiolytics). And these two phenomena, one by default and one by excess, perhaps should be reconsidered. It is for these reasons that I often think if it would not be more practical to name the drugs in another way, less categorical and more focused on the concrete reality of the cellular effects that these medications generate. I often like to refer to the so-called antipsychotics as dopamine blockers. Because what is common to all of them is that they block D2 dopamine receptors. To a greater or lesser extent, in a more or less irreversible way, with preference in one neuronal path or another (mesolimbic, mesocrotical, tuberoinfundibular or nigrostriatal) with more or less effects on serotoninergic or adrenergic receptors but in the end all of them are dopamine blockers. Naming them like this could change the "stigma" to which these medications are subject, antipsychotics that not only treat psychotic symptoms. The same goes for the SSRIs, which are antidepressants, but that are many other things: the common thing in them is that they have effects on serotonin, which invites me to call them sertoninergic modulators. In this way, when you prescribe it for an anxious condition, the patient would not ask you why you indicated an antidepressant without being depressed, or the primary care physician would not underuse them in obsessive or anxious conditions. Likewise, the so-called anxiolytic molecules could be called GABA modulators, because it is common to all these medications (modulate GABAergic transmission) and perhaps in this way, not by calling them anxiolytics, we could save a lot of benzodiazepine prescriptions that can later generate addiction problems. and that do not solve the anxious picture that underlies.

\section{Conclusion}

In summary, I believe that the names of psychiatric drug groups are not entirely practical and may require an in-depth review. 